\title{
HUMAN EMOTION ESTIMATION FROM EEG AND FACE USING STATISTICAL FEATURES AND SVM
}

\author{
Strahil Sokolov ${ }^{1}$, Yuliyan Velchev ${ }^{2}$, Svetla Radeva $^{3}$ and Dimitar Radev ${ }^{4}$ \\ ${ }^{1,3}$ Department of Information Technologies, \\ University of telecommunications and post, Sofia, Bulgaria \\ ${ }^{2,4}$ Department of Telecommunications, \\ University of telecommunications and post, Sofia, Bulgaria
}

\begin{abstract}
An approach is presented in this paper for automated estimation of human emotions from combination of multimodal data: electroencephalogram and facial images. The used EEG features are the Hjorth parameters calculated for theta, alpha, beta and gamma bands taken from pre-defined channels. For face emotion estimation PCA feature are selected. Classification is performed with support vector machines. Since the human emotions are modelled as combinations from physiological elements such as arousal, valence, dominance, liking, etc., these quantities are the classifier's outputs. The best achieved correct classification performance for EEG is about $76 \%$. Classifier combination is used to return the final score for the particular subject.
\end{abstract}

\section{KEYWORDS}

Multimodal human emotion, EEG, arousal, valence, BCI

\section{INTRODUCTION}

Analysis of human emotions from multimodal data is still a challenging task for the researchers worldwide. Since the early nineties of the past century the research on human emotions has brought together clinical researchers and engineers who have tried to automate this task. Facial analysis is one of the most popular techniques for automatic estimation of human emotions.

In recent years, multimodal approaches for human emotion estimation have emerged. Researches have tried to incorporate the human electroencephalogram (EEG) signals for emotional state analysis and this represents a challenging area of modern research. Historically EEF processing was considered a traditionally medical area and now it is being used for emotion analysis. Recent advances in the area of EEG analysis are said to deliver promising emotion recognition results already. There still seem to be gaps in terms of stability and accuracy in those algorithms. This is what motivated us to research in the area to provide a framework for reliable EEG emotional state estimation with main application in the Brain-Computer Interfaces (BCI) domain. The human emotion is a highly subjective phenomenon: it has been accepted by phsycologists that multiple dimensions or scales can be used to categorize emotions. A two-dimensional model of emotion is introduced in [1] (Fig. 1). The valence axis represents the quality of an emotion ranging from 
unpleasant to pleasant. The arousal axis refers to the quantitative activation level ranging from calm to excited state.

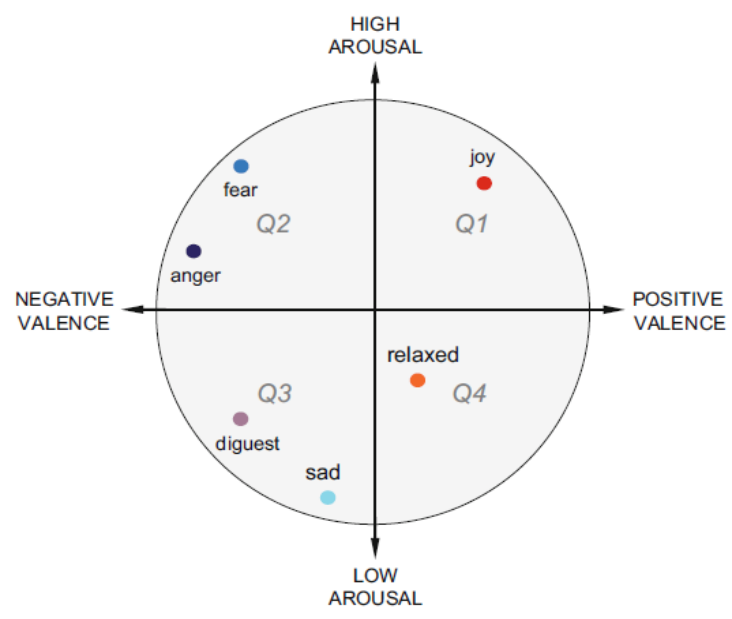

Figure 1. Two-dimensional human emotion model [2]

We have taken into consideration some of the most distinguished works in the area of EEG analysis. In [3] the authors used the EEG signal to classify two basic emotions: happiness and sadness. These emotions are evoked by showing subjects pictures that contain facial expressions of smile and cry. The authors propose a frequency band searching method to choose an optimal band into which the recorded EEG signal is filtered. They use Common Spatial Patterns (CSP) and linear Support Vector Machine (SVM) to classify these two emotions. To investigate the time resolution of classification, they explore two kinds of trials with lengths of $3 \mathrm{~s}$ and $1 \mathrm{~s}$. Classification accuracies of $93.5 \%$ and $93 \%$ are achieved on 10 subjects for $3 \mathrm{~s}$ and $1 \mathrm{~s}$ trials, respectively. Their experimental results indicate that the gamma band (roughly $10 \mathrm{~Hz}$ to $30 \mathrm{~Hz}$ ) is suitable for EEG-based emotion classification.

In [2] an approach is presented for EEG-based emotions recognition using time and frequency features. The time features are in fact some statistical quantities such as means and standard deviations of the raw signals and its first and second derivatives as well. The classified emotions are: joy, relax, sad and fear. The best accuracy is about $66 \%$ among three types of classifiers.

In [4] the achieved accuracy of the emotional valence is about $71 \%$. The technique relies on changes in the power spectrum of short-time stationary oscillatory EEG processes within the standard EEG frequency bands. The classification stage is logistic regression with elastic-net regularization. Another interesting approach is presented in [5]. The features are extracted from very limited set of electrodes and the dimensionality is further reduced with Principal Component Analysis (PCA). The performance is evaluated for arousal, valence and modality separately. As can be expected the arousal is with highest classification accuracy (over 90\%). A classification rate of $83.3 \%$ is reported in [6]. The authors have experimented with different classifiers and discrete wavelet transform as feature extraction technique. Very important results in terms of correlations between EEG waves activities from certain brain regions and the human emotions during a game play are published in [7]. These correlations are considered in the features extraction stage in our paper. In [8] is given an approach for emotions recognition using brain activity. The following types of features are used: EEG frequency band power, cross-correlation between EEG band powers, peak frequency in alpha band and Hjorth parameters.

The performances for classification in five classes is above $30 \%$. These results are indicative for a significant variability of EEG-based features for emotion recognition among different subjects. 
However, a reliable recognition of the extreme human emotions is still possible considering its distances in the two-dimensional model (Figure 1.).

Analysis of facial emotions has been in research for quite a while. There are a few works which are worth mentioning. The authors of [17] describe an approach using a combination of higherorder auto-correlation and fisher weights to classify emotions. The highest accuracy was with the combined method and reached about $97.9 \%$. In [13] an approach is presented where EEG and peripheral physiological signals from 12 participants had been recorded. The responses to emotional videos were classified with SVM into five classes namely, joy, sadness, disgust, fear, and relax. The achieved accuracy of $41.7 \%$ using EEG signals has been reported. Feature level combination of EEG signals and peripheral physiological signals failed to improve the classification accuracy.Combined or multimodal estimation of face emotions is a fairly new area of research. The authors of [15] propose the use of EEG and face analysis from video to detect emotion. Report on the accuracy has not been published. In [16] the authors combine EEG and eye gaze data to classify emotions. Based on single modality classification of arousal and valence in three classes, the authors obtained $62.1 \%$ and $50.5 \%$ accuracy eye gaze data. The authors proposed feature-level fusion and decision-level score fusion of the results and were able to improve accuracy to about $76 \%$ for arousal and $68.5 \%$ for valence.

The outline of the paper is organized as follows: in section 2 an overview is provided of the EEGbased emotion recognition including EEG features extraction, features selection and classification. In section 3 we provide details for the proposed approach for face data processing. In section 4 we describe the combination of classifier results. Section 5 describes the evaluation of EEG signals processing with corresponding emotional quantities, as well as the facial data processing and classifier combination. The last section discusses the results and marks out some aspects for future work.

\section{EEG EMOTION ESTIMATION}

The EEG-based human emotion recognition system is shown in Fig. 2. This paper does not cover the emotion model. As can be seen the dominance and liking are optional in the final emotion estimation.

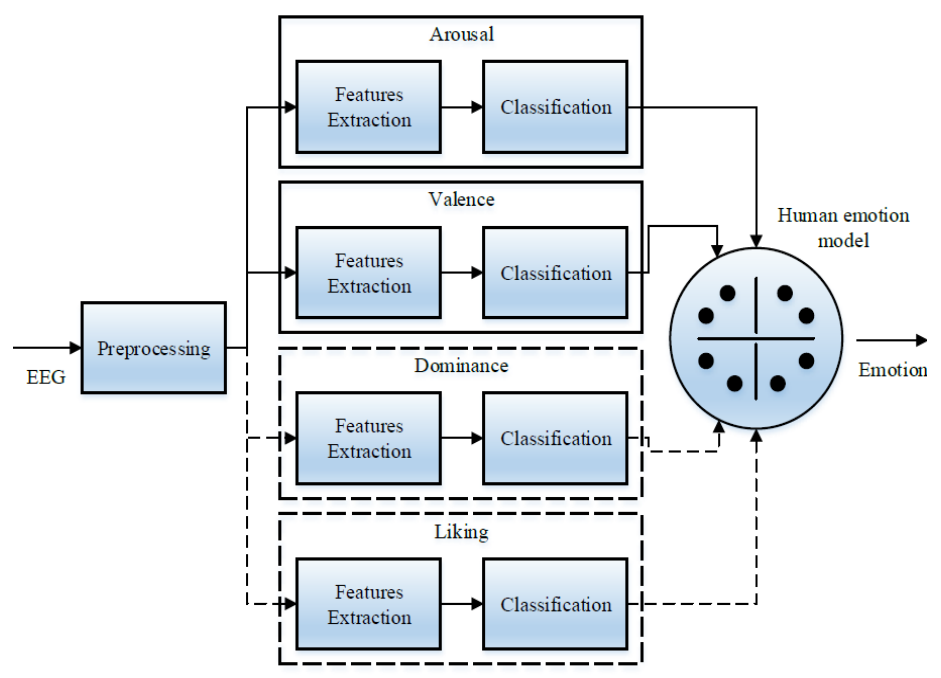

Figure 1. EEG-based human emotion recognition system 


\subsection{EEG Preprocessing}

We have increased the topographical localization of the EEG current sources using the scalp surface Laplacian spatial filtering. We have used the spherical spline method [9] that is the human head is modelled as a sphere. The spatial electrode coordinates are as suggested in the CSD toolbox implementation [9]. The parameter for spline flexibility is set to its default value of 4 .

\subsection{EEG Features Extraction}

Extraction of the features is realized via the EEG activities: $\theta$ (frequency range from $4 \mathrm{~Hz}$ to 7 $\mathrm{Hz}), \alpha(8 \mathrm{~Hz}$ to $13 \mathrm{~Hz}), \beta(14 \mathrm{~Hz}$ to $29 \mathrm{~Hz})$ and $\gamma(30 \mathrm{~Hz}$ to $45 \mathrm{~Hz})$. The used filter banks are realized as zero-phase Butterworth IIR.

EEG Channels Selection: Some researches confirm the positive valence and happy emotions are connected with an increase of frontal spectral coherence mainly in alpha band. Also the right parietal beta power increases. The higher arousal (excitation) causes an increase of coherence in parietal EEG and an increase of beta waves power. The alpha activity decreases as well. The emotion's strength is related to higher beta/alpha activity ratio in the frontal lobe. The beta activity at the parietal lobe is also increased. Considering the correlations found in [10] (Fig. 3) for arousal estimation we have used $\mathrm{CP} 6, \mathrm{Cz}, \mathrm{Fz}$ and $\mathrm{FC} 2$ electrodes. The chosen electrodes for valence estimation are: $\mathrm{Oz}, \mathrm{PO} 4, \mathrm{CP} 1, \mathrm{FC} 6, \mathrm{Cz}$ and $\mathrm{T} 8$.

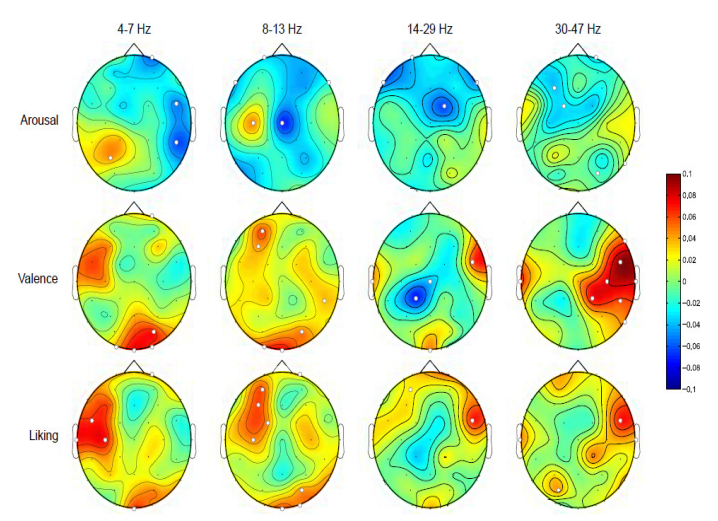

Figure 3. The mean correlations of the valence, arousal and general ratings with the power in the EEG waves [10]

1) The Feature Vectors: For a given EEG channel $c$ and band $b$ a feature vector is composed as follows:

$$
\mathbf{f}_{c, b}=\left[A c t_{c, b}, M_{o b}, b, C p l_{c, b}\right],
$$

where $A c t_{c, b}=\operatorname{var}\left(j_{c, b}(t)\right), \quad M o b_{c, b}=\sqrt{\frac{\operatorname{var}\left(\frac{d j_{c, b}}{d t}\right)}{A c t_{c, b}}}$ and $C p l_{c, b}=\sqrt{\frac{\operatorname{var}\left(\frac{d^{2} j_{c, b}}{d t^{2}}\right)}{\operatorname{var}\left(\frac{j_{c, b}}{d t}\right)}-M o b_{c, b}^{2}}$ are the Hjorth parameters [11] (known as activity, mobility and complexity). For arousal estimation the feature vector is organized as augmentation of $\mathbf{f}_{C P 6, \theta}, \mathbf{f}_{C z, \alpha}, \mathbf{f}_{F C 2, \beta}$ and $\frac{A c t_{F z, \beta}}{A c t_{F z, \alpha}}$. For valence estimation the feature vector consists of $\mathbf{f}_{O z, \theta}, \mathbf{f}_{P O 4, \alpha}, \mathbf{f}_{C P 1, \beta}, \mathbf{f}_{F C 6, \gamma}, \mathbf{f}_{O z, \beta}, \mathbf{f}_{C z, \beta}, \mathbf{f}_{T 8, \gamma}$ and $\mathbf{f}_{F C 6, \gamma}$ 


\subsection{EEG Features Selection}

Among many methods for features selection, we have chosen the popular Minimum Redundancy and Maximum Relevance (mRMR) criterion [12]. The relevance $R L$ of the set of selected features $F=\left\{f_{1}, f_{2}, \ldots\right\}$ and target classes $C$ is:

$$
R L=\frac{1}{|F|} \sum_{f_{i} \in F} I\left(f_{i}, C\right),
$$

where $I$ denotes the mutual information. The redundancy $R D$ of the features is:

$$
R D=\frac{1}{|F|^{2}} \sum_{f_{i}, f_{j} \in F} I\left(f_{i}, f_{j}\right) .
$$

For incremental search $\max [I(F, C)]$ is equivalent to $\max [R L(F, C)-R D(F)][12]$.

Our second suggestion for features is mRMR selection from all possible sets of ratios $\frac{A c t_{c, b}}{A c t_{c, b}}, c \neq k$ and $\frac{M o b_{c, b}}{M o b_{k, b}}, c \neq k$ where $c$ and $k$ denote the EEG channel and $b$ is the activity $(\theta, \alpha$ or $\beta)$.

\subsection{Classification}

Since the features for arousal and valence are very different, we have trained two classifiers of type SVM. We suggest classifying only the prime emotional quantities (arousal, valence, etc.). The emotions can be further inferred using and appropriate emotion model (two or threedimensional) (Figure 2.).

\section{FACIAL EMOTION ESTIMATION}

\subsection{Data acquisition and preprocessing}

The proposed approach for face detection and validation is based on our previous research [19]. It utilizes the OpenCV face detection algorithm [18] and a convolutional neural network.

The cascade structure of the face is intended for higher speeds of image processing due to the fast background rejection and focus on face-like regions. But in comparison with the monolithic classifiers, the cascade classifier (for example, Haar-like features' cascade of weak classifiers [18]) increases the detection error and false alarms rate. Therefore, it is necessary to join the rapid cascaded classifier with the accurate monolithic one within the two-level combined cascade of classifiers instead of using them independently. This is realized in order to achieve higher detection and lower false alarm rates. The two-level cascade of classifiers is called "combined" since it combines different types of classifiers, which have been proved in the course of time: the first level is represented by the Haar-like features' cascade of weak classifiers, which is responsible for the face-like objects detection, and the second level is a convolutional neural network for the objects' verification (Figure 4) 


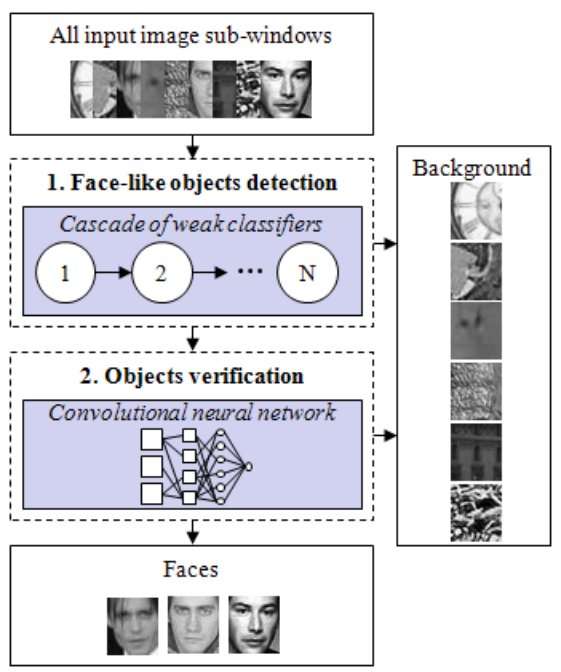

Figure 4. Face detection process using combined cascade of neural network classifiers

In this phase the fairly fast face detector is also able to deliver faces in frontal pose. This depends on the training set of images for the CNN. In our approach this has proven to be useful since we are using short-length videos of the subjects' faces may have slight fluctuations off the frontal pose.

\subsection{Facial features extraction}

The usage of PCA is proposed for facial features extraction. PCA has the property of packing the greatest energy into the least number of principal components. It computes the basis of a space which is represented by the training images as vectors. These basis vectors are computed by solution of an eigenproblem, and as such the basis vectors are eigenvectors. These eigenvectors are defined in the image space. They can be treated as images and indeed resemble faces. Hence they are usually referred to as eigenfaces. The components of the projected images in the eigenspace are characterised by statistical independence.

PCA is a linear transformation of type $\mathbf{Y}=\mathbf{W}^{\mathrm{T}} \mathbf{X} . \mathbf{X}$ is the matrix of the normalized faces, extracted from the video of the person; each face image is represented as a row vector. The major step in PCA is determining the basis $\mathbf{W}$. This basis is calculated by solving the problem of eigenvalues and eigenvectors of covariance matrix of $\mathbf{X}$ :

$$
\mathbf{C}_{\mathbf{X}}=E\left\{(\mathbf{X}-\overline{\mathbf{X}})(\mathbf{X}-\overline{\mathbf{X}})^{\mathbf{T}}\right\}
$$

$\overline{\mathbf{X}}$ is a matrix with each row representing the average of all faces, the so called mean face. The solution of the eigenvalue problem is stated as:

$$
\mathbf{C}_{\mathbf{X}} \mathbf{W}=\mathbf{W} \Lambda
$$

Each of the columns of $\mathbf{W}$ represents a normalized eigenvector of $\mathbf{C}_{\mathbf{X}}$ and $\Lambda_{\text {is a diagonal }}$ matrix with the eigenvalues on the main diagonal. Solving (5) can be done by finding roots of characteristic polynomials or by Jacobi rotation. Dimensionality reduction can be achieved by first rearranging eigenvalues in descending order and then rearranging $\mathbf{W}$ to match $\Lambda$. Next, the dimension of the eigenspace is calculated by the criteria normalized Residual Mean Square Error: 


$$
\operatorname{RMSE}(b)=\sum_{i=1}^{b} \lambda_{i} / \sum_{i=1}^{p} \lambda_{i}>T
$$

where $T$ is a threshold representing the fraction of the power of the signal, that must be preserved in the output space. Dimensionality reduction is an intuitive approach, since there exists a lot of statistical redundancy in natural images. Using the reduced covariance matrix is another possibility for reducing dimensionality even before resorting to (6).

\section{COMbining ClaSSIFIER RESUltS}

The combined classification that we propose is expected to increase accuracy and reduce noise using two parallel modules (distributed system). The output of the combined-modality biometric systems of type Multi-Sample-Multi-Source is the average score form the outputs of both modalities according to [20]:

$$
y_{\text {combined }}=\frac{1}{M} \sum_{j=1}^{M}\left[\frac{1}{N} \sum_{i=1}^{N}\left(y_{i, j}\right)\right] .
$$

where $y_{\text {combined }}$ is the combined output score, $M$ is the number of modalities used (EEG and Face), $N$ is the number of samples for each biometric modality, $y_{i, j}$ is the output of the system for the $i$-th sample from $j$-th modality and $y_{i, j} \in[0, \ldots, 1]$.

\section{EXPERIMENTAL RESULTS}

\subsection{The Used Dataset}

For our experiments we have used the DEAP dataset [10]. It consists of multimodal data physiological including EEG signals taken from 32 leads. Each subject participates with 40 trials with duration of $60 \mathrm{~s}$. The EEG signals were downsampled to $128 \mathrm{~Hz}$, bandpass filtered $(4 \mathrm{~Hz}$ to $45 \mathrm{~Hz}$ ) and the EOG artefacts were removed as suggested in [10]. The data was averaged to the common reference.

\subsection{Performance Evaluation}

We have used the accuracy as criterion for classification performance:

$$
A c c=\frac{T P+T N}{P+N}
$$

where $T P$ denotes the number of true positives, $T N$ is the number of true negatives. The denominator in (8) denotes the total population of positives $P$ and negatives $N$.

The performance is validated and evaluated using the $\mathrm{k}$-fold technique. That is, a testing part is extracted from the whole dataset. The rest of the dataset is used to train the classifier. This procedure repeats (10 times in our case) and the accuracy is calculated as an average of the accuracies in the iterations. The arousal and valence scores in dataset are given as fractional numbers ranging from 1 to 9 . We have quantized these scores to 3,5 and 7 levels and the testing was performed for each case. The accuracies of the estimated arousal are given in Table 1. Respectively the results for valence are summarized in Table. II. 
Table 1. Classification Performance for Arousal with Feature Vectors Built According to (8)

\begin{tabular}{|l|l|}
\hline Quantization Levels & Accuracy, \% \\
\hline 3 & 77.5 \\
\hline 5 & 63.2 \\
\hline 7 & 34.5 \\
\hline
\end{tabular}

Table 2. Classification Performance for Valence with Feature Vectors Built According to (1)

\begin{tabular}{|l|l|}
\hline Quantization Levels & Accuracy, \% \\
\hline 3 & 75.2 \\
\hline 5 & 57.2 \\
\hline 7 & 25.5 \\
\hline
\end{tabular}

As an alternative some experiments have been performed using the ratios for activities and mobilities for theta, alpha and beta waves. In this case the features have been selected using the mRMR method. The first 20 features for arousal and valence are given in Table. III. In Fig. 4 can be seen the calculated classification accuracies versus dimensionality of the feature vectors. Not all possible sets of features have been tested since the number of combinations is too large for an average computational machine.

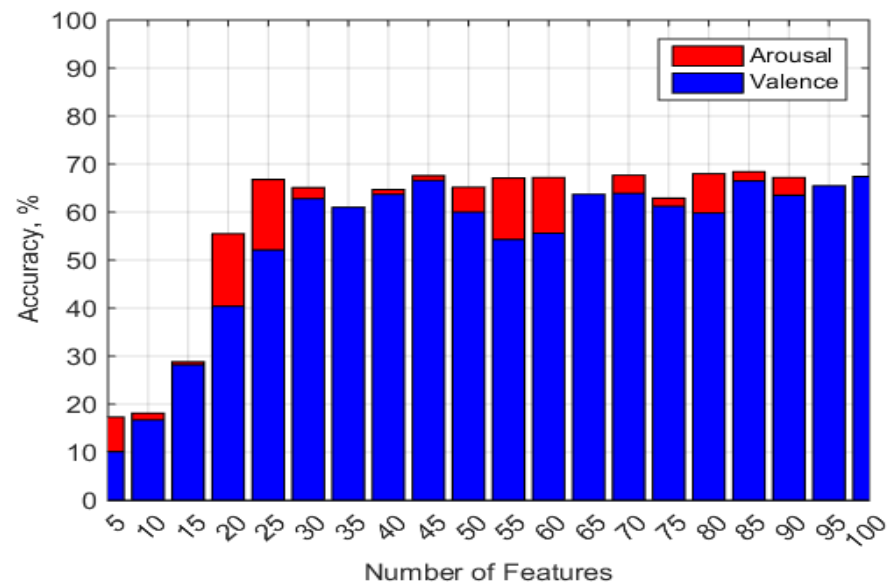

Figure 4. Classification accuracies for arousal and valence versus dimensionality of the feature vectors. The feature selection is according to $\mathrm{mRMR}$ method

Table 3. The 20 Most Discriminative Features for Arousal and Valence

\begin{tabular}{|l|l|l|}
\hline Index & Arousal & Valence \\
\hline 1 & $\frac{A c t_{F p 2, \theta}}{A c t_{C 4, \beta}}$ & $\frac{A c t_{F p 2, \theta}}{A c t_{C 4, \beta}}$ \\
\hline 2 & $\frac{M o b_{F p 1, \theta}}{M o b_{F p 1, \alpha}}$ & $\frac{M o b_{F p 1, \theta}}{M o b_{F p 1, \alpha}}$ \\
\hline 3 & $\frac{M o b_{F p 1, \theta}}{M o b_{F p 1, \beta}}$ & $\frac{M o b_{F p 1, \theta}}{M o b_{F p 1, \beta}}$ \\
\hline 4 & $\frac{M o b_{F p 1, \theta}}{M o b_{A F 3, \alpha}}$ & $\frac{M o b_{F p 1, \theta}}{M o b_{A F 3, \alpha}}$ \\
\hline 5 & $\frac{M o b_{T 7, \alpha}}{M o b_{A F 4, \theta}}$ & $\frac{M o b_{F p 1, \theta}}{M o b_{A F 3, \beta}}$ \\
\hline 6 & $\frac{M o b_{F p 1, \theta}}{M o b_{A F 3, \beta}}$ & $\frac{M o b_{F p 1, \theta}}{M o b_{F 3, \alpha}}$ \\
\hline 7 & $\frac{A c t_{C 4, \beta}}{A c t_{P 4, \theta}}$ & $\frac{M o b_{F 7, \alpha}}{M o b_{O z, \theta}}$ \\
\hline 8 & $\frac{M o b_{F 7, \alpha}}{M o b_{T 7, \theta}}$ & $\frac{M o b_{F p 1, \theta}}{M o b_{F 3, \beta}}$ \\
\hline 9 & $\frac{M o b_{F p 1, \theta}}{M o b_{F 3, \alpha}}$ & $\frac{A c t_{O z, \theta}}{A c t_{A F 4, \theta}}$ \\
\hline
\end{tabular}




\begin{tabular}{|l|l|l|}
\hline 10 & $\frac{A c t_{C P 5, \theta}}{A c t_{F p 2, \theta}}$ & $\frac{M o b_{F p 1, \alpha}}{M o b_{P 8, \theta}}$ \\
\hline 11 & $\frac{M o b_{F p 1, \theta}}{M o b_{F 3, \beta}}$ & $\frac{M o b_{T 7, \alpha}}{M o b_{P 4, \theta}}$ \\
\hline 12 & $\frac{M o b_{F p 1, \theta}}{M o b_{F 7, \alpha}}$ & $\frac{A c t_{C P 5, \theta}}{A c t_{F p 2, \beta}}$ \\
\hline 13 & $\frac{A c t_{F C 1, \alpha}}{A c t_{T 8, \theta}}$ & $\frac{M o b_{F p 2, \alpha}}{M o b_{F 4, \theta}}$ \\
\hline 14 & $\frac{A c t_{P O 3, \beta}}{A c t_{P z, \theta}}$ & $\frac{M o b_{F p 1, \theta}}{M o b_{F 7, \alpha}}$ \\
\hline 15 & $\frac{M o b_{F p 1, \theta}}{M o b_{F 7, \beta}}$ & $\frac{A c t_{F C 1, \beta}}{A c t_{P 4, \theta}}$ \\
\hline 16 & $\frac{M o b_{C z, \alpha}}{M o b_{T 8, \theta}}$ & $\frac{M o b_{F 7, \alpha}}{M o b_{C 3, \theta}}$ \\
\hline 17 & $\frac{A c t_{A F 3, \theta}}{A c t_{P z, \beta}}$ & $\frac{M o b_{P 3, \beta}}{M o b_{P z, \theta}}$ \\
\hline 18 & $\frac{M o b_{F p 1, \theta}}{M o b_{F C 5, \alpha}}$ & $\frac{M o b_{F p 1, \theta}}{M o b_{F 7, \beta}}$ \\
\hline 19 & $\frac{M o b_{C P 1, \alpha}}{M o b_{F 4, \theta}}$ & $\frac{A c t_{F p 1, \theta}}{A c t_{P z, \beta}}$ \\
\hline 20 & $\frac{M o b_{F p 1, \theta}}{M o b_{F C 5, \beta}}$ & $\frac{M o b_{F p 1, \theta}}{M o b_{F C 5, \alpha}}$ \\
\hline
\end{tabular}

An exact comparison of accuracies between our approach and those achieved by other researchers is not possible because of the different testing dataset. Also the chosen outputs of the classifiers are not the same (different levels of quantization for arousal and valence). Nevertheless, an approximate comparison can be seen in Table 4 .

Considering the accuracies in this study and those published by other researchers, the practical use of EEG-based emotion recognition is limited in terms of distinguishing between some very basic emotional states such as joy, anger, sadness and relax. The channels selected using mRMR method in general do not match those suggested by the other studies. That confirms the poor subject-independence of the EEG features for emotions recognitions. Face emotion estimation runs in parallel and contributes to the improvement of the scores generated by the EEG analysis module. The proposed PCA-based face emotion estimation provides an additional improvement for the EEG signal analysis.

Table 4.Classification Performance of the Presented Approach Compared with Results of Related Works

\begin{tabular}{|l|l|}
\hline Approach & $\begin{array}{l}\text { Average accuracy for } \\
\text { arousal and valence, \% }\end{array}$ \\
\hline In [5] & 96.2 \\
\hline In [8] & 36.5 \\
\hline In [2] & 66.5 \\
\hline In [4] & 71.3 \\
\hline In this paper & 76.4 \\
\hline
\end{tabular}

\section{CONCLUSIONS}

This work presented an approach for automated multimodal EEG and face-based estimation of human emotions. The accuracy was investigated for different levels of EEG arousal and valence. For EEG, the maximal accuracy of $76.4 \%$ is achieved when the arousal and valence is classified in only three levels. The improvement of the classification is delivered via parallel face emotion analysis system based on PCA. The classifiers that we have used are SVM and we propose scorelevel decision fusion. In our future work we will seek to implement future improvement of the BCI which we are developing. We are considering the usage of Active Appearance Models as well as 3D facial emotion recognition as further improvement in order to achieve our objective and create $\mathrm{HCI}$ for people with motor disabilities. 


\section{ACKNOWLEDGEMENTS}

This work is part of a research project funded by the National Scientific Research Fund at the Bulgarian Ministry of Education and Science DFNI I02/2014 "Human-computer interface for medical assisting systems for life improving of people with propelling problems".

\section{REFERENCES}

[1] R. Davidson, G. Schwartz, C. Saron, J. Bennett, and D. Goleman, "Frontal versus parietal eeg asymmetry during positive and negative affect," Psychophysiology, vol. 16 (2), pp. 202-203, 1979.

[2] X.-W. Wang, D. Nie, and B.-L. Lu, "EEG-Based Emotion Recognition Using Frequency Domain Features and Support Vector Machines," ICONIP'11 Proceedings of the 18th international conference on Neural Information Processing, vol. Part I, pp. 734-743, 2011.

[3] M. Li and B.-L. Lu, "Emotion classification based on gamma-band EEG," Engineering in Medicine and Biology Society, 2009. EMBC 2009. Annual International Conference of the IEEE, pp. 12231226, 2009.

[4] C. Kothe, S. Makeig, M. Soleymani, and J. Onton, "Emotion Recognition from EEG During SelfPaced Emotional Imagery," 2013 Humaine Association Conference on Affective Computing and Intelligent Interaction (ACII), pp. 855-858, 2013.

[5] D. Oude Bos, "EEG-based emotion recognition-The Influence of Visual and Auditory Stimuli," Capita Selecta (MSc course), 2006.

[6] M. Murugappan, N. Ramachandran, and Y. Sazali, "Classification of human emotion from EEG using discrete wavelet transform,” J. Biomedical Science and Engineering, vol. 3, pp. 390-396, 2010.

[7] B. Reuderink, C. Mühl, and M. Poel, "Valence, arousal and dominance in the EEG during game play," International Journal of Autonomous and Adaptive Communications Systems, vol. 6 (1), pp. 44-62, 2013.

[8] R. Horlings, D. Datcu, and L. J. M. Rothkrantz, "Emotion Recognition using Brain Activity," International Conference on Computer Systems and Technologies-CompSysTech'08, vol. II, pp. 1-6, 2008.

[9] J. Kayser and C. Tenke, "Principal components analysis of Laplacian waveforms as a generic method for identifying ERP generator patterns: I. Evaluation with auditory oddball tasks," Clinical Neurophysiology, vol. 117 (2), pp. 348-368, 2006.

[10] S. Koelstra, C. Mühl, M. Soleymani, J.-S. Lee, A. Yazdani, T. Ebrahimi, T. Pun, A. Nijholt, and I. Patras, "DEAP: A Database for Emotion Analysis using Physiological Signals," IEEE Transactions on Affectiv Computing, vol. 3 (1), pp. 18-31, 2012.

[11] B. Hjorth, "EEG analysis based on time domain properties," Electroencephalography and Clinical Neurophysiology, vol. 29 (3), p. 306-310, 1970.

[12] H. Peng, F. Long, and C. Ding, "Feature Selection Based on Mutual Information: Criteria of MaxDependency, Max-Relevance, and Min-Redundancy," IEEE Transactions on Pattern Analysis and Machine Intelligence, vol. 27 (8), pp. 1226-1238, 2005.

[13] Takahashi, Kazuhiko. "Remarks on SVM-based emotion recognition from multi-modal bio-potential signals." In Robot and Human Interactive Communication, 2004. ROMAN 2004. 13th IEEE International Workshop on, pp. 95-100. IEEE, 2004. 
[14] T. Kanade, J.F. Cohn, and Y. Tian. Comprehensive database for facial expression analysis. In Proceedings of the fourth IEEE International conference on automatic face and gesture recognition (FG'00), pp. 46-53, Grenoble, France,2000.

[15] SAVRAN, Arman, et al. Emotion Detection in the Loop from Brain Signals and Facial Images. In: Proceedings of the eNTERFACE 2006 Workshop. 2006.

[16] Soleymani, M., Pantic, M. and Pun, T., 2012. Multimodal emotion recognition in response to videos. IEEE transactions on affective computing, 3(2), pp.211-223.

[17] Shinohara, Yusuke, and N. Otsuf. "Facial expression recognition using fisher weight maps." In Automatic Face and Gesture Recognition, 2004. Proceedings. Sixth IEEE International Conference on, pp. 499-504. IEEE, 2004

[18] Viola, Paul, and Michael J. Jones. "Robust real-time face detection." International journal of computer vision 57, no. 2 (2004): 137-154.

[19] Paliy, Ihor, Anatoly Sachenko, Yuriy Kurylyak, Ognian Boumbarov, and Strahil Sokolov. "Combined approach to face detection for biometric identification systems." In Intelligent Data Acquisition and Advanced Computing Systems: Technology and Applications, 2009. IDAACS 2009. IEEE International Workshop on, pp. 425-429. IEEE, 2009.

[20] Poh, N., Bengio, S., Korczak, J., A Multi-sample Multi-source Model for Biometric Authentication. Proceedings of the 2002 12th IEEE Workshop on Neural Networks for Signal Processing, 375-384.

\section{AUTHORS}

Strahil Sokolov, $\mathrm{PhD}$ is an assistant professor at the Department of Information technologies, University of Telecommunications and Post -Sofia

Yuliyan Velchev, is $\mathrm{PhD}$ at the Department of Telecommunications, University of Telecommunications and Post -Sofia.

Prof. Svetla Radeva, DSc, PhD, is full professor at the Department of Information technologies, University of Telecommunications and Post -Sofia.

Prof. Dimitar Radev, DSc, PhD, is full professor at the Department of Telecommunications, University of Telecommunications and Post -Sofia.
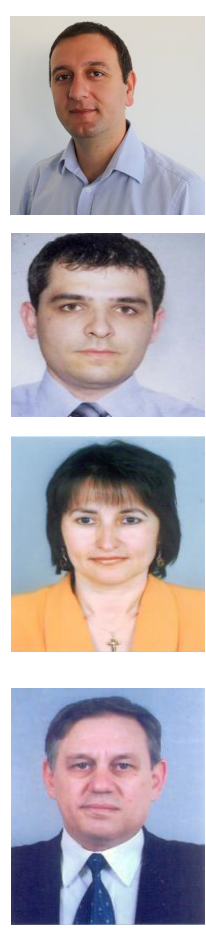